\title{
ESTUDIO GEOLÓGICO - MINERO Y GEOECONÓMICO PRELIMINAR DE UN YACIMIENTO DE SÍLICE (CUARCITA) EN CANDARAVE
}

\author{
Responsable: Ing. Gualberto Tejada Bedoya \\ Miembro: Ing. Carlos Huisa Ccori \\ Miembro: Ing. Zenón Sarmiento Mejia \\ Miembro: Ing. Rómulo Cerdeña Vélez
}

\begin{abstract}
RESUMEN
Se ha realizado un estudio detallado de la geología, la cubicación de reservas probadas y probables del yacimiento de sílice (cuarcita). En la industria minera un campo atractivo para aplicar las opciones reales y aprovechar las ventajas que se precisan es cuando existe una mayor incertidumbre en la valoración o tasación de yacimientos o activos mineros que está cambiando con fuerza la nueva ecuación de negocios. La incertidumbre de los resultados posibles y futuros de cada periodo de la ulterior explotación se basa en estimar un plan minero adecuado, desarrollar un plan de negocio inteligente que permita obtener una viabilidad del proyecto en plazo, costo, calidad y riesgos controlados y, además, cuantificar apropiadamente los factores críticos de éxito que afectan la generación de valor del activo minero que se está evaluando. Ante estas perspectivas es que se plantea este estudio de pre - factibilidad de explotación del yacimiento de Silica (cuarcita) en la provincia de Candarave.
\end{abstract}

\begin{abstract}
A detailed study of Geology has been made, the cubing of proven and probable reserves of the Silica deposit (Quartzite). In the mining industry an attractive field to apply the real options and to take advantage of the advantages that need is when a greater uncertainty in the valuation or mining appraisal of deposits or assets exist, that this changing with force the new equation of businesses. The uncertainty of the possible and future results of every period of the later operation is based on considering a suitable mining plan, to develop a plan of intelligent business that allows to obtain a viability of the project in term, cost, controlled quality and risks, and, in addition, appropriately to quantify the critical factors of success that affect the generation of value of the mining assets that this evaluating itself. Before these perspective it is that this study of Pre considers - feasibility of operation of the deposit of Silicifies (Quartzite) in the province of Candarave.
\end{abstract}

\section{UBICACIÓN}

La zona de afloramiento de la cuarcita se encuentra ubicada a 220 kilómetros de la ciudad de Tacna, geológicamente en el flanco andino, es la entidad topográfica que se extiende al norte y noreste de las pampas costaneras, es un territorio escabroso y muy disectado que se desarrolla entre 2000 y $4000 \mathrm{~m}$ de altitud. El afloramiento abarca una superficie de 100 hectáreas, en el anexo de Marjani, departamento de Tacna, provincia de Candarave, distrito de Quilahuani en las siguientes coordenadas U.T.M.

$\begin{array}{ll}8087000 & 370000 \\ 8086000 & 370000 \\ 8086000 & 369000 \\ 8087000 & 369000\end{array}$

Con una altitud aproximada de 2980 m.s.n.m.

\section{ACCESIBILIDAD}

La via de acceso desde la ciudad de Tacna se realiza por la carretera parcialmente asfaltada Tacna - Tarata (tramo de $145 \mathrm{~km}$ ), para continuar por la carretera afirmada Tarata-Candarave, hasta pasar por centro poblado menor Buena Vista ( $\mathrm{km} 58)$, luego se ingresa por una trocha carrozable hasta un tramo de aproximadamente $17 \mathrm{~km}$ y se arriba a Marjani. Asimismo existe otra via de acceso desde la ciudad de Moquegua mediante la carretera binacional Ilo-Desaguadero, totalmente asfaltada, hasta inmediaciones del poblado de Huaitire (a $105 \mathrm{~km}$ de Moquegua), en donde existe un desvío de donde parte una carretera afirmada hasta la localidad de Candarave (tramo de $70 \mathrm{~km}$ ) para luego proseguir hasta el centro poblado menor de Aricota, posteriormente continuar hasta el anexo de Marjani, a donde se ingresa por medio del cauce de la quebrada Cañaveral.

\section{FISIOGRAFÍA}

\section{Relieve y Altitudes}

Un rasgo físico interesante en la zona es la existencia de la laguna de Aricota, ubicada en las cabeceras del rio Curibaya, constituyendo un nivel de base local para los ríos Callazas y Salado que descienden a lo largo de valles poco profundos, de suave pendiente y sección transversal a subrectangular, labrados en los tufos y clásticos de las formaciones Huaylillas y Capillune.

En cambio el valle del rio Curibaya que nace 
de Aricota está integramente labrado en los volcánicos Toquepala, se caracteriza por su gran profundidad, alrededor de $1000 \mathrm{~m}$, fuerte gradiente y sección transversal en " $\mathrm{V}$ ", por lo que existe una marcada diferencia morfológica entre los valles situados aguas arriba de la laguna y el valle Curibaya, diferencia que se atribuye al control litológico y al nivel de base de la laguna de Aricota.

Se aprecia entre la cadena volcánica y el flanco disectado por el sur, un rasgo fisiográfico caracterizado por la presencia de una superficie suavemente ondulada que se levanta desde los 3600 hasta lo $4000 \mathrm{~m}$ de altitud. Otro rasgo orográfico es la del volcán Yucamane formado durante el Terciario Superior y Cuaternario $(5508 \mathrm{~m})$, conservando casi intacta su forma cónica visible desde distancias considerables.

\section{Geomorfología}

La zona de estudio geomorfológicamante se ubica al suroeste del volcán Yucamane, sobre un frente de flujos de piroclásticos (tobas) que por erosión forman mesetas disectadas por quebradas, formando laderas abruptas en la margen izquierda del río Callazas. Localmente las laderas presentan pendientes entre moderadas $\left(30^{\circ}\right)$, abruptas (verticales), a suaves en el fondo del valle donde se ubica actualmente el poblado de Marjani. Antes del sismo del 23 de junio del 2001 que afectó gran parte del sur peruano. En el Área afloran rocas sedimentarias del tipo areniscas y cuarcitas grises, violáceas, bastante fracturadas y alteradas, dispuestas en bancos medianos a gruesos. Sobre estas rocas yacen rocas volcánicas del tipo ignimbritas de color ocre rojizas, soldadas y poco soldadas, bastante fracturadas. Estas rocas se hallan parcialmente cubiertas de depósitos aluviales, residuales y coluviales.

\section{Hidrología}

Los ríos Callazos y Salado drenan la zona, sus nacientes se encuentran en la cadena volcánica, se forman por una serie de pequeños tributarios cuyas fuentes de alimentación son las lluvias estaciónales, los deshielos de los nevados y los bofedales, sus cursos siguen una orientación norte-sur a lo largo de los valles angostos hasta desembocar en la laguna de Aricota.

\section{Clima y Vegetación}

El clima es templado a frio por su altitud, el periodo de lluvias se extiende de diciembre a marzo, produciendo un alto incremento del caudal del río Callazas, como producto de las fuertes lluvias que caen en la Cordillera de los Andes, siendo afectadas por desbordes terrenos de cultivos localizados en las riberas del rio Callazas,inmediaciones del poblado Marjani. El clima caracteristico de esta zona es frío con precipitaciones sólidas, la acción del viento desde moderada a fuerte en diferentes épocas del año, las temperaturas durante las noches descienden a varios grados bajo cero. La agricultura está reducida a la explotación de los terrenos que existen a lo largo del fondo o piso de los valles, se cultiva papas, maíz, habas, ajo, cebolla, orégano, etc.

\section{Geologia Local}

La litologia y estructura de la zona es variada, va desde el mesozoico hasta los volcánicos recientes, asi como la presencia de grandes fallas tectónicas, algunas activas. El anfiteatro de Marjani es el asiento del pueblo del mismo nombre y de pequeñas campiñas que se localizan en las partes abiertas del valle.

En la quebrada Marjani, que se encuentra a $700 \mathrm{~m}$ al norte del pueblo de Aricota, se ha observado la presencia, muy local, de $150 \mathrm{~m}$ de arenisca cuarzosa de color gris blanquecino a marrón, tiene un buzamiento de $40^{\circ}-25^{\circ}$ al noreste variable y con presencia de gran plegamiento; el afloramiento en estudio está considerado como una arenisca cuarzosa que pertenece al Grupo Yura y que comprende la Formación Puente (Caloviano) seguida de la Formación Cachios de rocas principalmente lutáceas, la Formación Labra de areniscas cuarzosas, cuarcitas y lutitas, seguida de la Formación Gramadal, constituida por calizas con fauna del Jurásico-Cretácico, y la Formación Hualhuani, con cuarcitas, la que por su posición estratigráfica corresponde al Cretácico. En el caso de la zona de estudio la que aflora es la formación Labra.

Suprayaciendo a esta formación se encuentra el volcánico Huaylillas que tiene una edad de 16,8 y 14,8 M.A. del Mioceno medio y que abarca gran parte del anfiteatro que envuelve al pueblo de Marjani.

Luego se encuentra el Grupo Barroso, que concierne al volcán Yucamane que para el caso está conformado por tobas y brechas, se le asigna una edad de 7,2 a 0,7 M.A. Un rango del Mioceno superior a Pleistoceno.

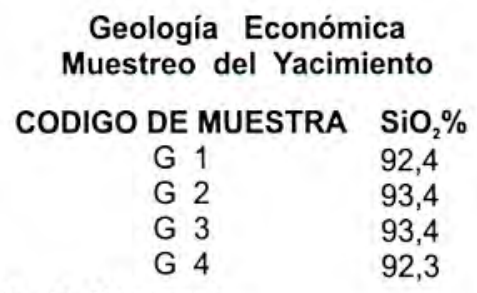

\section{Cálculo de Reservas}

Se ha procedido a ejecutar el cálculo de reservas con un Software denominado Data Geosis 2.0, habiéndose obtenido un volumen de 
5418689 metros cúbicos, que multiplicado por su peso específico de la cuarcita se tiene como reserva total de la zona en estudio 14630460 toneladas métricas.

\section{PLANEAMIENTOY MÉTODO DE EXPLOTACIÓN}

\section{Diseño y Planeamiento de Minado}

\section{Definición}

Los yacimientos se encuentran en la naturaleza en diferentes formas, posiciones y profundidades. Para ubicarlos y cuantificarlos existen poderosas herramientas Software, entre las más conocidas están: Gemcom, Data Mine, Linux, Whitle, etc. En nuestro caso se ha utilizado el Software denominado Data Geosis 2.0, el cual nos ayudara para determinar las reservas y la cubicación del yacimiento. Los parámetros más utilizados en estos programas son:

- Forma y posición del yacimiento.

- Valor económico del yacimiento.

- Tipo de formación geológica y calidad del terreno.

- Relación de desmonte mineral (Stripping Ratio).

- Topografía del terreno superficial.

\begin{tabular}{|l|l|l|l|l|}
\hline \multirow{2}{*}{ Nivel } & \multicolumn{2}{|c|}{ Silice (Si O2) } & \multirow{2}{*}{ Desmonte $(\mathrm{t})$} & \multirow{2}{*}{ TOTAL $(\mathrm{t})$} \\
\cline { 2 - 3 } & Mineral $(\mathrm{t})$ & Ley & & \\
\hline 3075 & - &.-- & 18475,00 & 18475,00 \\
\hline 3070 & 117831,04 & 92,4 & 78554,03 & 196385,07 \\
\hline 3065 & 231234,67 & 93,4 & 154156,45 & 385391,12 \\
\hline 3060 & 572464,94 & 93,4 & 381643,29 & 954108,23 \\
\hline 3055 & 2910160,09 & 92,4 & 1940106,73 & 4850266,82 \\
\hline 3050 & 4946585,44 & 93,2 & 3297723,62 & 8244309,06 \\
\hline & 8778276,18 & 92,9 & 5870659,12 & 14648935,30 \\
\hline
\end{tabular}

\section{Reservas Minables}

\section{Planeamiento a Mediano y Largo Plazo}

\section{Parámetros:}

Inicio de explotación (desbroce) 2006

Altura de banco (explotación) $5 \mathrm{~m}$.

Densidad del mineral $\quad 2,7 \mathrm{t} / \mathrm{m}^{3}$

Densidad del desmonte $\quad 2,4 \mathrm{t} / \mathrm{m}^{3}$.

Desbroce desmonte $38475 \mathrm{t}$.

Explotación venta a SPCC 100 t/día

Días trabajados 25 días al mes

Tonelaje de producción mineral 30000 t/año

Tonelaje de desmonte $\quad 15000$ t/año

\section{Método de Explotación}

\section{Explotación Tipo Canteras (Teoria):}

\section{a) Definición}

Las canteras son bastante similares a las minas a cielo abierto, y el equipo empleado es el mismo. La diferencia es que los materiales extraidos suelen ser minerales industriales y materiales de construcción. En general, casi todo el material que se obtiene de la cantera se transforma en algún producto, por lo que hay bastante menos material de desecho. Asu vez, esto significa que al final de la vida útil de la cantera queda una gran excavación. No obstante, debido a los bajos precios que suelen tener los productos de la mayoría de las canteras, éstas tienen que estar situadas relativamente cerca de los mercados. $\mathrm{Si}$ no fuera así, los gastos de transporte podrian hacer que la cantera no fuera rentable.

\section{Costos de Producción}

Costos de Producción Mensual

\begin{tabular}{|c|c|c|c|c|}
\hline \multicolumn{5}{|c|}{ Tonelaje Explotada al Mes $=3750 \mathrm{t}$. } \\
\hline $\begin{array}{l}\text { I. AREA } \\
\text { ADMINISTRATIVA }\end{array}$ & Costo Mes \$ & $\$ / t$ & Sub-Total \$/t & $\begin{array}{l}\text { Total } \\
\text { Costo \$/t. }\end{array}$ \\
\hline \multicolumn{5}{|l|}{ 1.- Personal de Oficina } \\
\hline Personal Administrativo & 338,24 & 0,09 & & \\
\hline Cargas Sociales & 35,53 & 0,009 & & \\
\hline Beneficios Sociales & 89,26 & 0,024 & 0,123 & \\
\hline 2.-Gastos Administrativos & 161,76 & 0.043 & 0,043 & 0,166 \\
\hline \multicolumn{5}{|c|}{ \| ÁREA DE PRODUCCIÓN } \\
\hline \multicolumn{5}{|l|}{ 1.- Personal Mina } \\
\hline Personal Mina & 1829 & 0,487 & & \\
\hline Cargas Sociales & 272 & 0,073 & & \\
\hline Beneficios Sociales & 611,56 & 0,163 & & \\
\hline Otros Varios & 29,41 & 0,008 & & \\
\hline \multirow{2}{*}{\multicolumn{5}{|c|}{$\begin{array}{l}\text { 2.- Gastos de } \\
\text { Operación }\end{array}$}} \\
\hline & & & & \\
\hline Explosivos (Anfo, Ful & 661,30 & 0,176 & & \\
\hline \multicolumn{5}{|l|}{ Minante, Guia) } \\
\hline Kerosene y Otros & 35,29 & 0,009 & & \\
\hline Alquiler de Maquinaria & 264,70 & 0.071 & & \\
\hline Mantenimiento & 29.41 & 0.008 & & \\
\hline \multicolumn{5}{|l|}{ Herram. } \\
\hline Botiquin & 5,88 & 0,001 & & \\
\hline Equipo de Trabaio & 29,41 & 0,008 & & \\
\hline \multicolumn{5}{|l|}{$\frac{(\text { Cascos }}{\text { Guantes, Zapatos etc) }}$} \\
\hline \multicolumn{5}{|l|}{ Guantes, Zapatos etc) } \\
\hline Subtotal & 1025,99 & & 0,273 & \\
\hline $\begin{array}{l}\text { 3.- } \text { Compromisos } \\
\text { Ambiental }\end{array}$ & 29,41 & 0,008 & 0,008 & 0,281 \\
\hline \multicolumn{5}{|c|}{ III AREA DE COMERCIALIZACIONN } \\
\hline 1.-Transporte & 11911.76 & 3.176 & & \\
\hline 2.- Pesado & 176,47 & 0.047 & & \\
\hline 3.- Descargas & 235.29 & 0.063 & & \\
\hline 4.- Peajes & 229.41 & 0.061 & & 3.347 \\
\hline IV ÁREA DE ASESORIA & Y TRIBUTOS & & & \\
\hline 1.- Asesor Minero & 58,82 & 0,016 & & \\
\hline 2.- Asesor de Explosivos & 23.53 & 0.006 & & \\
\hline 3.-Vigencia y etros & 29,41 & 0,008 & & \\
\hline 4.-Impuestos a la Renta & 330.88 & 0.088 & & \\
\hline $\begin{array}{l}\text { 5.- Otros no } \\
\text { Comtemplados }\end{array}$ & 14.70 & 0.004 & & 0.122 \\
\hline
\end{tabular}

COSTO TOTAL DE PRODUCCIÓN TOTAL \$ / 4,647

\section{EVALUACIÓN DEL PROYECTO}

\section{Ingresos}

Se consideran ingresos a la captación de dinero por venta de la silice durante 10 años .el precio actual del silice es de 41 nuevos soles con una ley de $90 \%$ de $\mathrm{SiO}_{2}$ y con una impureza menor de $3 \%$ de aluminio. Existen castigos y premios de s/ 2,20 por punto hasta $87 \%$ de ley.

Para el proyecto se consideran tres alternativas de precios: pesimista \$9/ton UK, realista \$11/ton UK, optimista \$12/ ton UK.

$$
\begin{aligned}
& \text { T= tonelada }=1000 \mathrm{~kg} . \\
& \text { Ton UK = tonelada larga } 2240 \text { libras } \\
& \text { Ton USA = tonelada corta } 2000 \text { libras }
\end{aligned}
$$

Se proyecta una producción mensual de $\mathrm{SiO}_{2}$ de 30000 ton UK/año con una ley promedio de $92,9 \%$ de $\mathrm{SiO}_{2}$. 
Para costo de producción se necesitan $\$ 17$ 432,77 al mes durante tres meses hasta poder vender el producto; entonces, el capital de trabajo necesario es de $\$ 52289,51$. Para asuntos de evaluación redondeamos a $\$ 52500$. Con estos datos ahora proyectamos las ventas para 10 años.

\section{Precio pesimista $\$ 9 /$ ton UK}

Según el análisis de sensibilidad del proyecto, se determinó lo siguiente:

\section{Análisis Financiero}

Realista precio a 11 \$/tel flujo de caja es $30143,57 \$$ Pesimista precio a 9 /t el flujo de caja es - $20562,41 \$$ Optimista precio a $12 \$ /$ tel flujo de caja es $42206,40 \$$

\section{Análisis Económico}

Realista precio a $11 \$ / t$ el flujo de caja es $44596,60 \$$ Pesimista precio a $9 \$ /$ el flujo de caja es $13263,12 \$$ Optimista precio a $12 \$ /$ el flujo de caja es $56659,44 \$$

Finalmente, se llega a la conclusión de que el proyecto es rentable según las condiciones planteadas para su explotación.

\section{CONCLUSIONES}

1. Las rocas más antigua que afloran en la zona de estudio son las areniscas cuarzosas del Grupo Yura, de Jurásico Superior, en las proximidades del pueblo de Marjani.

2. Las reservas probadas y probables del yacimiento es mineral sílice 8778276,18 toneladas con una ley promedio de $92,9 \%$ y como desmonte 5870 659,12 toneladas.

3. El método de explotación más adecuado se ha planteado a Tajo Abierto tipo cantera con bancos de 5 metros de altura y bermas de $2,5 \mathrm{~m}$. El proceso de operación unitaria será con voladuras masivas (calambuco), carguío y transporte en camiones hacia la planta de fundición de SPCC.

4. El costo total de producción para la explotación del yacimiento asciende a $4,647 \$ /$ t.

5. Según el análisis económico de sensibilidad se llegó a los siguientes datos:

\section{Análisis Financiero:}

- Precio realistaa $11 \$ / t$ resulta un flujo de caja $3143,57 \$$

- Precio pesimista a $09 \$ /$ resulta un flujo de caja $20562,41 \$$

- Precio optimistaa12\$/t resulta un flujo de caja 42206,40\$

Análisis Económico:

- Precio realista a $11 \$ / t$ resulta un flujo de caja 44596,60\$

- Precio pesimista a $09 \$ /$ resulta un flujo de caja $13263,12 \$$

- Precio optimista a 12 \$/t.resulta un flujo de caja 56 659,44\$

En conclusión general, el proyecto es rentable y optimista para su explotación.

\section{BIBLIOGRAFÍA}

Bateman Alan M. Yacimientos minerales de rendimiento económico.

Cornelius S. Hurlbut. Manual de mineralogia de Dana.Ed. Reverte 1974.

Corrales, C. Estratigrafía. Editorial Rueda, Madrid 1977.

EMJ Operating Handbook of Mineral Processing 1974.

Frederic H. Lahee. Geologia práctica. Ediciones Omega, 1970.

Klaws Stainmüller. Depósitos metálicos en el Perú, INGEMMET, 1999.

Kraus Hunt Romsdell.Mineralogía. McGraw-Hill. Krumbein Y Sloss. Estratigrafia y sedimentación. Editorial Uthea.

\section{AFLORAMIENTO DEL YACIMIENTO DE SILICA (CUARCITA)FOTO ORIENTACIÓN AL NW}

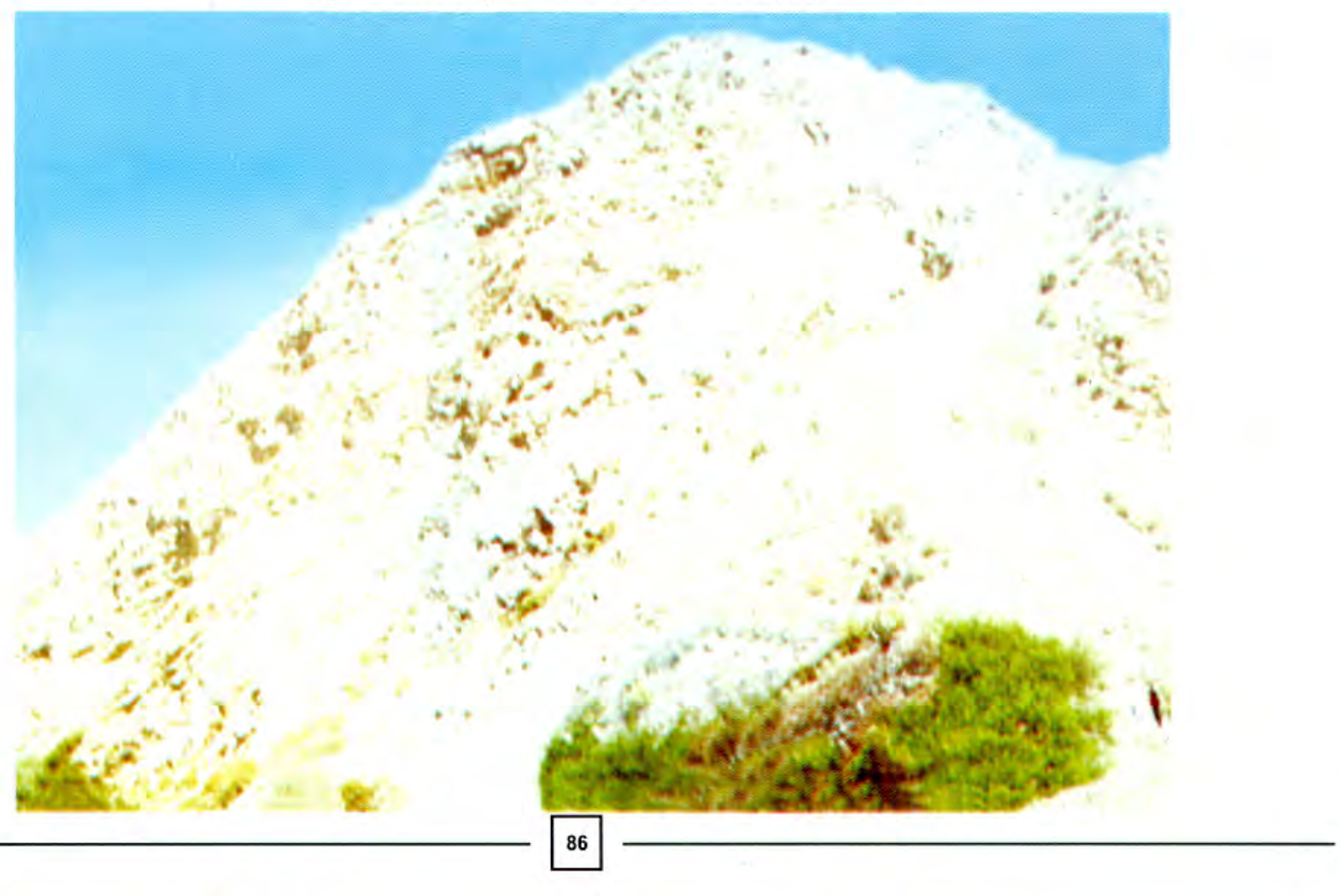

